\title{
Reconstruction of In-cylinder Temperature, Equivalence Ratio and NOx Distribution Fields using Proper Orthogonal Decomposition Technique
}

\author{
H. Akbari ${ }^{1}$, A. Salavati-Zadeh ${ }^{2 \dagger}$, A. Javaheri ${ }^{2}$, V. Esfahanian ${ }^{3}$, S.V. Ghavami ${ }^{1}$ and H. \\ Ghomashi $^{4}$ \\ ${ }^{I}$ Senior Researcher, Vehicle, fuel and Environment Research Institute, University of Tehran, North Kargar \\ Ave., Tehran, Iran. \\ ${ }^{2}$ Senior Researcher, Ph.D., Vehicle, fuel and Environment Research Institute, University of Tehran, North \\ Kargar Ave., Tehran, Iran. \\ ${ }^{3}$ Professor, School of Mechanical Engineering, University of Tehran, North Kargar Ave., Tehran, Iran. \\ 4 Assistant Professor, Faculty of Engineering, Islamic Azad University, South Tehran Branch, Ahang Ave., \\ Tehran, Iran.
}

†Corresponding Author Email: alisalavati@ut.ac.ir

(Received October 20, 2015; accepted December 10, 2015)

\begin{abstract}
The present study aims to reduce the computational cost of in-cylinder phenomena simulation under the light of employing Proper Orthogonal Decomposition (POD) technique. The equivalence ratio as the main identifier for soot formation tendency along with temperature and nitrogen oxide fields, are studied inside a gas-fuelled engine. The required correlation matrix is built based on ten snapshots obtained from the results of engine three-dimensional simulation, which are verified based on experimental data. The AVL-FIRE v.2013 software is used to carry out the three dimensional simulations. The flow field at $3250 \mathrm{rpm}$ is then estimated by POD coefficients and subsequent curve fittings. To validate the reduced order results, this condition is simulated by the software. For instance, temperature and equivalence ratio fields at top dead center and 5 degree after top dead center are compared. The relevance index for equivalence ratio indicates about $96 \%$ consistency between reduced order and $3 \mathrm{D}$ simulation results. On the other hand, this index is found to be about $99 \%$ at both crank angels for temperature, which proves a more coherent structure in the temperature field than that of equivalence ratio. Meanwhile, the analysis of 3D simulation results by POD demonstrates a more coherent structure for the in-cylinder flow regime at top dead center. This consistency is obtained in spite of computation time of POD being approximately $1 \%$ of $3 \mathrm{D}$ simulation time.
\end{abstract}

Keywords: Internal combustion engine; Simulation, Proper orthogonal decomposition.

\section{NOMENCLATURE}

$\begin{array}{ll}\mathrm{a}^{(\mathrm{k})} & \text { POD modes corresponding coefficients } \\ \text { ATDC } & \text { after top dead center } \\ \text { CFD } & \text { computational fluid dynamics } \\ \text { CR } & \text { compression ratio } \\ \mathrm{e}_{\mathrm{i}} & \text { node } \mathrm{i} \text { energy content } \\ \text { ECFM } & \text { extended coherent flame model } \\ \text { EVC } & \text { exhaust valve close } \\ \text { EVO } & \text { exhaust valve open } \\ \mathrm{F}^{(\mathrm{k})} & \text { kth snapshot }\end{array}$

\section{INTRODUCTION}

Today, the engine designers are facing one of the most challenging problems, the simultaneous

$\begin{array}{ll}\text { IVC } & \text { intake valve close } \\ \text { IVO } & \text { intake valve open } \\ \text { PIV } & \text { particle image velocimetry } \\ \text { POD } & \text { proper orthogonal decomposition } \\ \mathrm{q} & \text { considered variable for POD analysis } \\ \mathrm{R}_{\mathrm{p}} & \text { relevance index } \\ \text { RPM } & \text { revolutions per minute } \\ \varphi^{(\mathrm{k})} & \text { POD modes }\end{array}$

reduction in the amount of soot particles and nitrogen oxides emitted from the engine. Developing different prototypes and performing costly experiments are no more feasible, since the regulatory agenda requires rapid responses. Despite 
the fact that employing numerical simulation has obviously paved the way through minimizing the amount of required experiments for the design process, it comes with shortcomings. Performing detailed simulations are time consuming and may cause high computational cost. Utilizing reduced order modeling techniques can therefore be a promising choice. Concerning this growing interest to decrease the simulation computational time and cost, lots of researches cope with engine model reduction. These attempts mainly include employing zero-dimensional e.g. (Rakopoulos, 2003) and quasi-dimensional, e.g. (Salavati-Zadeh et al., 2012), along with using empirical correlations, e.g. (Rakopoulos, 2003), single step chemistry, e.g. (Graciano, 2015) and reduced chemical schemes, e.g. (Chen et al., 2009) for emission formation. Other choices may be the modelling approaches and the strategies which follow the general lines of orthogonal transformation, among which Proper Orthogonal decomposition (POD hereafter) is proved to be an efficient and favorable tools for reduction of large datasets to small number of scalar coefficients.

POD technique is based on the methodology proposed by Karhunen (1946) and Loeve (1946), known as K-L decomposition. This approach has then undergone various developments and was named POD algorithm in simulation of turbulent flows by Lumley (1967). Vast improvements were done after the development of snapshot technique (Sirovich, 1987) and caused serious reduction in the amount of numerical integrations.

Fogleman et al. (2004) first employed this methodology for simulation of internal combustion engines. They studied tumble breakdown by applying this procedure on the velocity field data gathered by Particle Image Velocimetry (PIV hereafter) technique. Under the light of the studying the POD first modes during intake and exhaust strokes, they concluded that considering the lower tumble breakdown during the intake stroke, the structure of the velocity field is more coherent. In addition, using the coefficients representing each mode, the cycle-by-cycle variation was investigated. Druault et al. (2005) by interpolating the coefficients corresponding to each POD mode obtained based on PIV, calculated the flow field in a different crank angle with high accuracy. Cosadia et al. $(2006,2007)$ investigated the swirl in a diesel engine cylinder in different cycles with reliable accuracy. By comparing the mean values of velocity field data and the field reconstructed by the POD first mode, they concluded that considering the high energy of the first mode, these two fields are identical and the other modes show the cycleby-cycle variation. Druault and Chaillou (2007) implemented this methodology to 3D flow fields and obtained cyclic variation by analyzing standard deviation of coefficients corresponding to POD modes. Imberdis (2009) compared the in-cylinder velocity field obtained by different turbulence models using POD technique. In this research, the POD modes were used for evaluation of the field's spatial differences, and the corresponding coefficients were utilized for cyclic variation estimation. Bizon et al. (2009, 2010) divided the field to mean flow and fluctuations. By using third and fourth moments of the data gathered from images, they splitted the fluctuations field into coherent and incoherent parts. They introduced the coherent part as the fluctuations in all the cycles and incoherent part as cycle-by-cycle variation. Chen et al. (2011) employed this technique to analyze 237 consecutive working cycles to investigate the combustion quality inside a direct injection engine.

It could be concluded that employing POD technique in engine mostly deal with analyzing the flow field and finding the fluctuations in different consecutive cycles with respect to time and space, considering other factors constant. In spite of this, using this technique for intermittent parameters such as engine speed, engine load and many other parameters could be a great advantage since detailed simulation of all operating conditions is not feasible or even possible anymore. To this end, Salavati-Zadeh et al. (2013) employed POD technique for simulation of the effects of cold mass flow rate and equivalence ratio on the formation of soot in laminar premixed flames.

The present study aims to investigate the applicability of POD technique for reducing the number of simulations required to assess the effect of engine speed on equivalence ratio as the soot formation tendency criteria and NOx formation along with temperature distribution field. A gasfuelled SI engine is chosen considering the availability of experimental setup. The required correlation matrix is built based on ten snapshots obtained from the results of engine threedimensional simulation, which are verified based on experimental data. The AVL-FIRE v.2013 software is used to carry out the three dimensional simulations. The flow field at $3250 \mathrm{rpm}$ is then estimated by POD coefficients and subsequent curve fittings. To validate the reduced order results, this condition is simulated by the software. The results prove this technique to be a promising tool to lower the computational cost.

\section{EXPERIMENTAL SETUP}

An AVL single cylinder test bed including a variable $\mathrm{CR}$ single cylinder spark ignition gasfueled engine (Fig. 1) is used as the experimental facility. The engine general specifications are listed in table 1. Despite the fact that the engine CR can be varied between 6 and 16, the case of 12 for CR is considered in this research. The experimental setup has previously been described in details by Javaheri et al. (2014).

\section{PROPER DECOMPOSITION \\ ORTHOGONAL}

The technique of Proper Orthogonal Decomposition is founded on the patterns generated by the simulation results or the experimental observations. The data are collected through manipulations of the 
Table 1 Single cylinder engine specifications (Javaheri et al., 2014)

\begin{tabular}{|c|c|}
\hline Bore & $86 \mathrm{~mm}$ \\
\hline Stroke & $86 \mathrm{~mm}$ \\
\hline Displacement Volume & $499.6 \mathrm{cc}$ \\
\hline CR & 12 \\
\hline Pin Offset & $0.6 \mathrm{~mm}$ \\
\hline No. of Valves & 4 \\
\hline Connecting Rod & $143 \mathrm{~mm}$ \\
\hline Ignition Timing & 19 BTDC \\
\hline IVO & 34 BTDC \\
\hline IVC & 126 BTDC \\
\hline EVO & 106 ATDC \\
\hline EVC & 14 ATDC \\
\hline
\end{tabular}

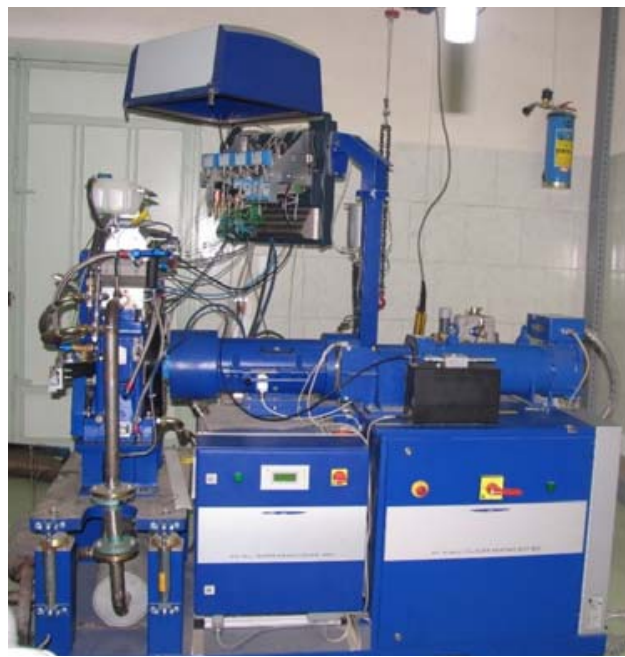

Fig. 1: Single Cylinder Test Bed (Javaheri et al., 2014).

process variables and disturbances. Since the excitation of a physical process is rarely random, the collected data usually demonstrates regularities. If a variable changes, a certain pattern keeps repeating itself from one condition to the next one. The fundamental idea behind this technique is to decompose the data into a linear combination of $M$ spatial basis functions or the POD modes, $\varphi^{(k)}$ and the corresponding coefficients, $a^{(k)}$.

$$
F^{(k)}(x, q)=\sum_{i=1}^{M}\left(a_{(i)}^{(k)}(q) \cdot \phi_{(i)}(x)\right)
$$

Where $F^{(k)}$ is the snapshot and is the considered variable. These basis functions or the modes describe the spatial distribution patterns. Associated with each mode, there are corresponding coefficients which are dependent on considered variable and demonstrate the consequence of its changes.

The commencement point of POD technique is a sequence of $\mathrm{M}$ instantaneous considered fields or snapshots. Each snapshot consists of quantity at $\mathrm{N}$ discrete locations in physical space. These locations are arbitrary, but they must be the same locations for all snapshots.

$$
F=\left[\begin{array}{cccc}
f_{l}^{(l)} & f_{2}^{(l)} & \ldots & f_{N}^{(l)} \\
f_{1}^{(2)} & f_{2}^{(2)} & \ldots & f_{N}^{(2)} \\
\vdots & \vdots & \ddots & \vdots \\
f_{1}^{(M)} & f_{2}^{(M)} & \ldots & f_{N}^{(M)}
\end{array}\right]
$$

The POD modes are obtained by solving the eigenvalue problem $C \psi=\lambda \psi . C$ is the correlation matrix:

$C=\frac{1}{N} F^{T} \cdot F$

A set of modes can be built by the eigenvectors:

$\phi_{(i)}(x)=\sum_{k=1}^{M} \psi_{i k} u_{k}$

where the index $k$ indicates the number of snapshots. The POD modes are independent of each other. In mathematical terminology, it means that they are mutually orthogonal. The coefficients associated with each mode are calculated by the projection of snapshots on the mode:

$a_{(i)}^{(k)}=\left\langle f^{(k)}, \varphi_{(i)}\right\rangle$

For the application of POD to define the importance of each mode, a criterion commonly used which is so-called mode energy criterion. In this criterion, the ratio between the mode energy and the whole energy of field is obtained. The mode energy, $e_{i}$, is defined by aggregate of coefficient squares associated to considered mode:

$e_{i}=\frac{1}{2} \sum_{k=1}^{K}\left(a_{i}^{(k)}\right)^{2}$

The energy fraction of $i$ th mode is given by

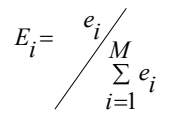

Furthermore, it can be shown that the magnitude of each eigenvalue is the same as twice the mean energy in the corresponding POD mode (Liu and Haworth, 2011).

It is beneficial to order the eigenvectors by descending magnitude of the eigenvalues, so that the first mode contains the highest energy. A sharp decrease in eigenvalue quantity with mode sequence would be a characteristic of a highly organized flow.

To have a visual comparison of the field distributions, a "relevance index $R_{p}$ " can be used to assess the similarity of any two distributions. This criterion obtained by projecting a distribution onto another one.

$R_{p}=\frac{\left(f^{1}, f^{2}\right)}{\left\|f^{1}\right\|\left\|f^{2}\right\|}$

Where the numerator denotes the inner product of 
two distributions and \|\| is the $l^{2}$ norm:

$$
\|f\|=(f, f)^{1 / 2}
$$

The numerical value of the relevance index varies from -1 to $1 . R_{p}=1$ means that the considered distributions are identical, while a value of -1 means that they are exactly opposite.

In this work, Proper Orthogonal Decomposition (POD) is coupled with curve fitting and employed to reconstruct information in between engine speeds taken from the AVL-FIRE v.2013 software.

In essence POD provides discernment of challenging phenomena in combustion that restrict the performance increments and reductions in fuel consumption and emissions. Understanding cycleto-cycle variations is a necessary first step toward controlling such variation.

\section{NUMERICAL SCHEME}

\subsection{Model description}

AVL-FIRE v.2013 is used to simulate a gas fuelled single cylinder spark ignition engine with the specifications listed in Tab. 1.

Table 1 single cylinder engine specifications (Javaheri et al. 2013).

\begin{tabular}{|c|c|}
\hline Bore & $86 \mathrm{~mm}$ \\
\hline Stroke & $86 \mathrm{~mm}$ \\
\hline Displacement & $499.6 \mathrm{cc}$ \\
\hline CR & 12 \\
\hline No. of Valves & 4 \\
\hline Connecting Rod & $143 \mathrm{~mm}$ \\
\hline Ignition Timing & $19^{\circ} \mathrm{BTDC}$ \\
\hline IVO & $34^{\circ} \mathrm{BTDC}$ \\
\hline IVC & $126^{\circ} \mathrm{BTDC}$ \\
\hline EVO & $106^{\circ} \mathrm{ATDC}$ \\
\hline EVC & $14^{\circ} \mathrm{ATDC}$ \\
\hline
\end{tabular}

The fixed wall boundaries, including liner, piston crown, valves, cylinder wall and exhaust port, are considered as isothermal. The Fame Engine Plus (FEP) Tool via AVL-FIRE was used to create moving mesh in the combustion chamber. The AVL CFD Solver is based on the finite volume discretization method. Semi-Implicit Method for Pressure-Linked Equations algorithm (SIMPLE) is used to the overall solution of the governing equations. The models used to simulate phenomena within the combustion chamber are given in Tab. 2 .

Table 2 Selection of models

\begin{tabular}{|l|c|}
\hline \multicolumn{1}{|c|}{ Models } & Values \\
\hline Turbulence & k-zeta-f \\
\hline Combustion & ECFM \\
\hline NO formation & xpanded Zeldovich Mechanism \\
\hline
\end{tabular}

\subsection{Model validation}

In order to assure the validity of the simulation results, the pressure history of experiment and numerical simulation at engine speed of $2000 \mathrm{rpm}$ are compared and illustrated in Fig. 1. The consistency between the results seems to be good and the root mean square of errors especially at the closed cycle period remains under $1.5 \%$.

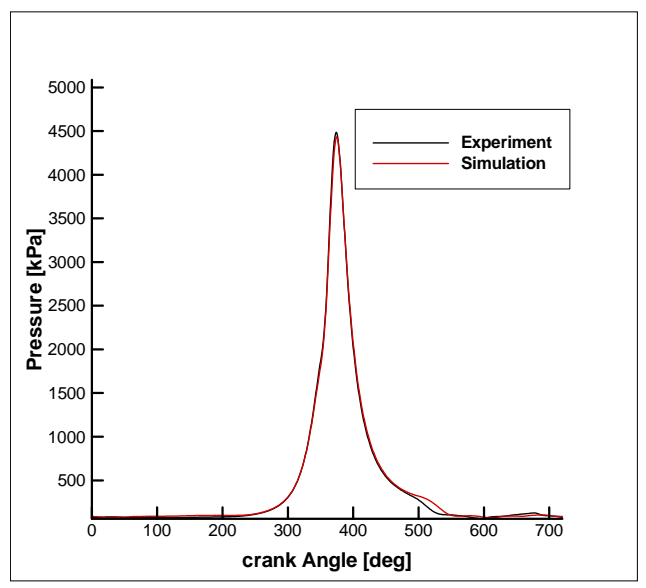

Fig. 1. Variation of in cylinder pressure with crank angle position

\section{NUMERICAL SCHEME}

In order to employ POD methodology, different snapshots were obtained by simulation of ten engines $(1000,1500,5500 \mathrm{rpm})$. The flow fields at $3250 \mathrm{rpm}$ are then estimated by POD coefficients and subsequent curve fittings. To validate the reduced order results, this condition is simulated by the software and the obtained temperature, equivalence ratio and $\mathrm{NOx}$ distribution fields at 5 degree after top dead center are compared.

\subsection{Equivalence ratio}

The equivalence ratio is chosen based on two important reasons. Equivalence ratio also plays role as one of the parameters with a considerable importance in second-law studies and combustion irreversibilities (Rakopoulos, 2005). In addition, the distribution of equivalence ratio defines as the main identifier of the soot formation zones and NOx distribution fields. At higher equivalence ratios, i.e., at richer conditions, considering the lower available oxygen the possibility of soot formation increases.

Fig. 2 demonstrates the cumulative energy of POD modes for equivalence ratio fields at top dead center and 5 degree after top dead center. The first mode at TDC contains larger percentage of total energy than $5^{\circ} \mathrm{ATDC}$. This indicates that the equivalence ratio field at TDC has a more coherent structure than the other crank angle. It should be noted that the mean flow at each condition is subtracted from the snapshots prior to accomplishing the POD technique. Therefore, one will be able to shed light on the fluctuations of considered field. This subtraction does not normally alter the form of the modes owing to the large energy associated with the mean flow repeated in each snapshot. 


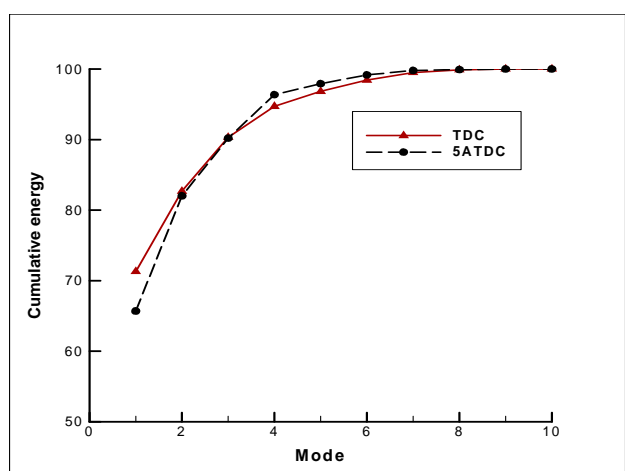

Fig. 2. Cumulative energy versus the number of the first POD mode for equivalence ratio results.

In order to estimate the equivalence ratio field at $5^{\circ} \mathrm{ATDC}$, the coefficients associated with the first mode at different speeds were plotted in Fig. 3. Subsecuently, the adequate polynominal equation can be obtained by curve fitting technique.

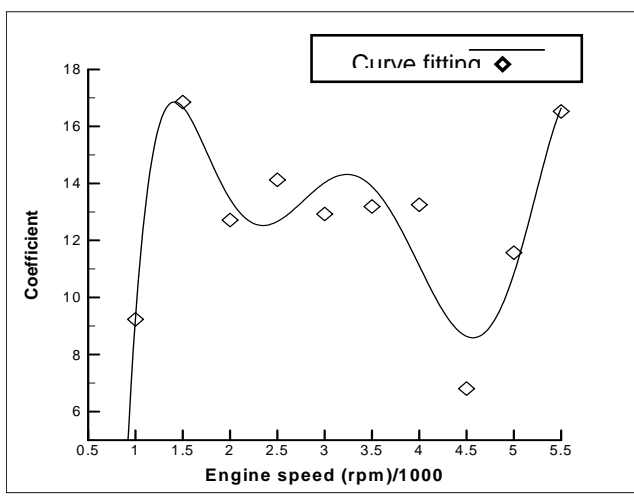

Fig. 3. the first mode coefficients related to engine speeds at $5^{\circ} \mathrm{ATDC}$.

$$
\begin{aligned}
y & =-0.3764 x^{6}+7.884 x^{5}-65.212 x^{4} \\
& +271.21 x^{3}-595.24 x^{2}+649.29 x-251.57
\end{aligned}
$$

By employing higher order equations, the more accurate curve fitting will be achieved. For instance, the 6th order polyniminal is sufficient for Fig. 3. By replacing 3.25 in equation 10 , the coefficient related to the first mode at 3250 RPM is obtained. This procedure is repeated for remained coefficients at considered speed. To construct the equivalence ratio field at 3250 RPM, the ten coefficients multiply by related modes that are spatial distributions and constant for whole speed. To validate the reduced order results, this condition is simulated by the software. Fig. 4 demonstrates the POD and software comparative data.

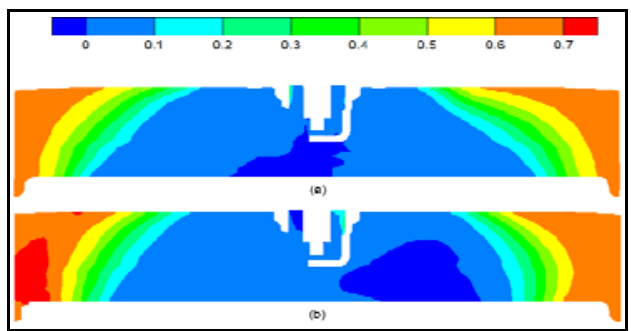

Fig. 4. Equivalence ratio fields at $5^{\circ} \mathrm{ATDC}(\mathrm{a})$ : software (b): POD.
Fig. 4 states the equivalence ratio fields on the same cutting plane including 2512 nodes. The relevance ratio is 0.967 , which shows a remarkable similarity between two results.

\subsection{Temperature distribution}

The heat flux to combustion chamber walls varies according to the operating conditions. Moreover, the heat flux to the different parts of the combustion chamber is not equal. Therefore, due to this nonuniform heat flux, the temperature distribution between locations on the combustion chamber is nonuniform. Furthermore, temperature distribution plays crucial roles in the spray and flame modelling (Heywood, 1988)

Fig. 5 shows the cumulative energy of POD modes for temperature fields at TDC and $5^{\circ} \mathrm{ATDC}$.

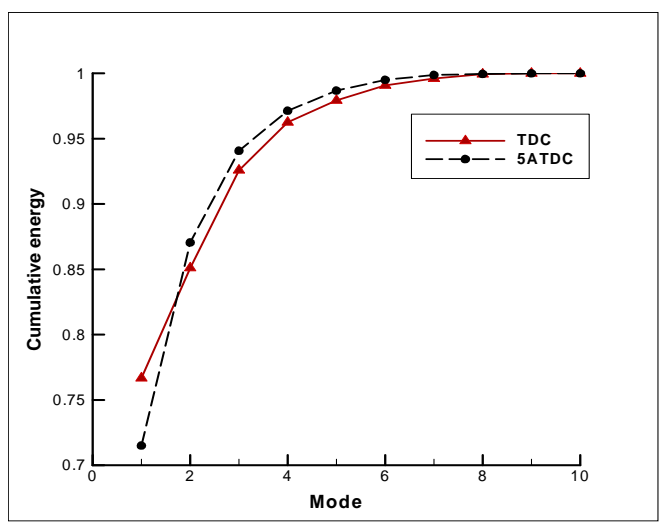

Fig. 5. cumulative energy versus the number of first mode for temperature distribution.

With regard to the high energy related to the modes it can be proved that temperature fields have more organized structures than equivalence tario fields. Moreover, Fig. 5 demonstrates a more coherent structure for the in-cylinder temperature regime at $5^{\circ} \mathrm{ATDC}$.

Fig. 6 shows the coefficients related to the engine speeds for the first 3 modes.

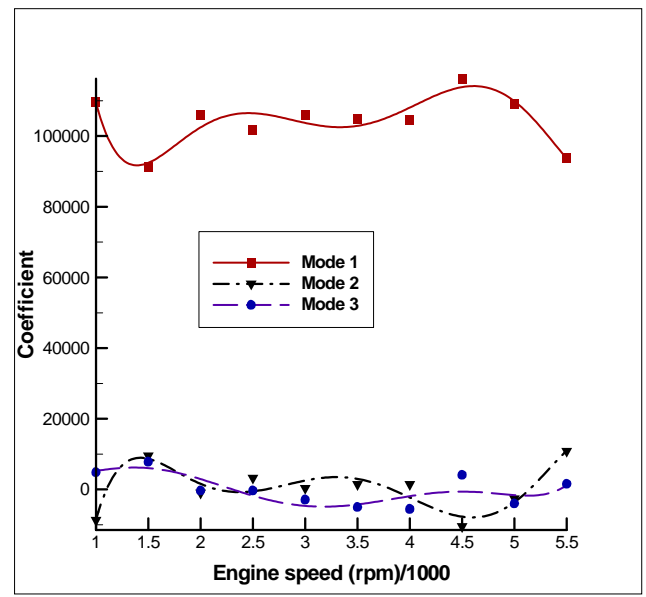

Fig. 6. the first 3 modes coefficients related to engine speeds at $5^{\circ} \mathrm{ATDC}$. 
As it is shown in Fig. 6, the quantity of coefficients related to the first mode is more considerable than other modes. Moreover, the significant difference between the first mode energy and others (Fig.5) describes the major role of this mode to reconstruct the fields or predict new conditions.

The comparison between the fields obtained from software and POD is shown in Fig. 7. The relevance ratio between two cutting planes is 0.998 .

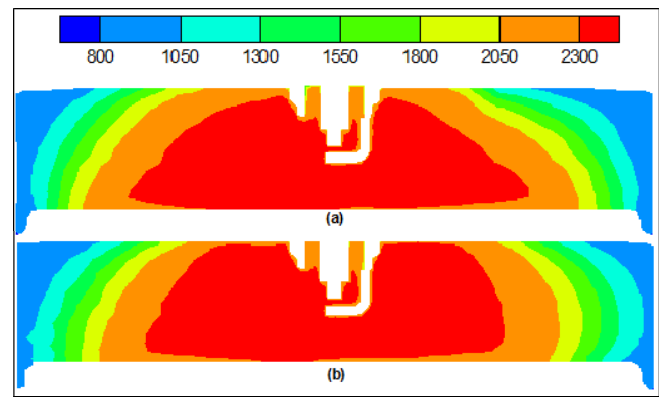

Fig. 7. Temperature distributions at $5^{\circ} \mathrm{ATDC}$ (a): software (b): POD.

\subsection{NO Mass Fraction}

The spark-ignition engine exhaust gases contain oxides of nitrogen, carbon monoxide and unburned hydrocarbons. Nitrogen oxide (NO) has a dominant amount between oxides of nitrogen. In addition to the temperature, the formation of $\mathrm{NO}$ depends on pressure, air-fuel ratio and location within the combustion chamber (Pulkrabek, 1997).

After employing POD method to the data set, it is proven that NO field at TDC has a more coherent structure than $5^{\circ} \mathrm{ATDC}$. (Fig. 8)

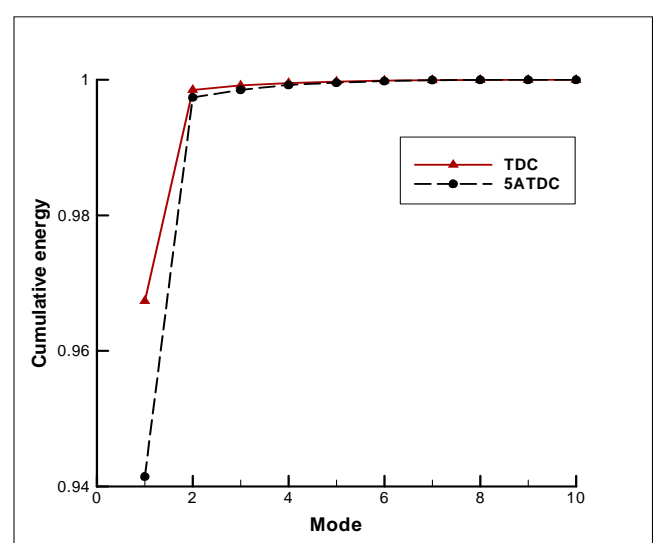

Fig. 8. cumulative energy versus the number of the first mode for NO field.

Due to the high energy of the first mode in Fig. 8, it appears that the first mode is adequate to predict a new condition. Consequently, only the coefficient related to the first mode is obtained.

Fig. 10 demonstrates the POD and software results for NO mass fraction. The relevance index is 0.962 .

\section{CONClusions}

In the present work, proper orthogonal

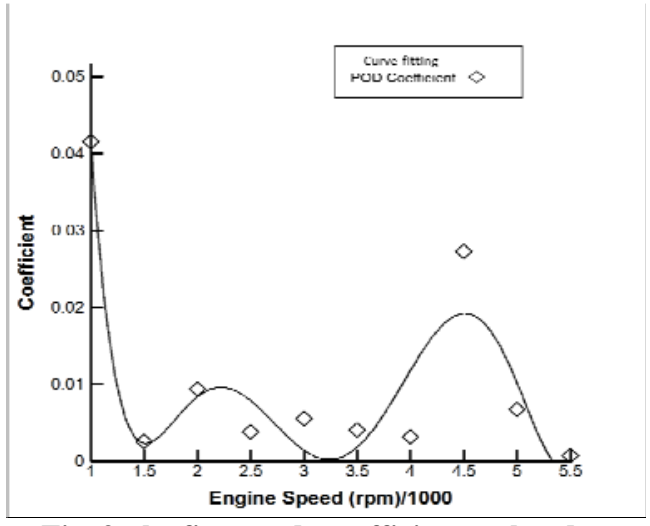

Fig. 9. the first mode coefficients related to engine speeds at $5^{\circ} \mathrm{ATDC}$ for NO mass fraction.

decomposition has been used for estimation of phenomena within the combustion chamber of single cylinder gas fuelled SI engine at compression

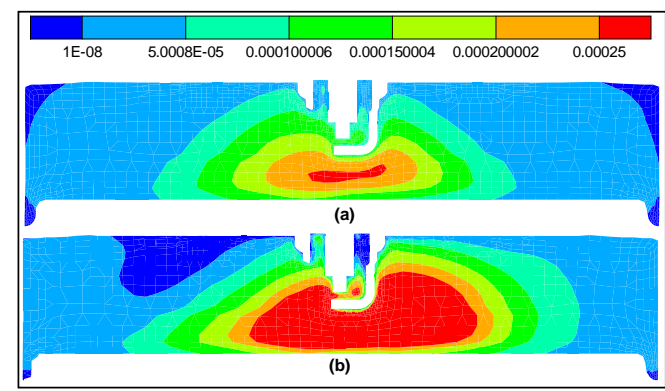

Fig. 10. NO mass fraction at $5^{\circ} \mathrm{ATDC}(\mathrm{a})$ : software (b): POD.

ratio of 12 based on results obtained from AVLFIRE v.2013 software. To validate the numerical results, the calculated pressure curve in $2000 \mathrm{rpm}$ is compared with the corresponding experimental and showed good agreement.

The correlation matrix is built based on simulation results for 10 different engine speeds. The flow field at $3250 \mathrm{rpm}$ is then estimated by POD coefficients and subsequent curve fittings. To validate the reduced order results, this condition is simulated by the software. For instance, temperature and equivalence ratio fields at top dead center and 5 degree after top dead center are compared. The relevance index for both parameters at both conditions is more than $95 \%$ indicating that employing POD could play a fruitful role.

This consistency is obtained in spite of computation time of POD being approximately $1 \%$ of $3 \mathrm{D}$ simulation time. The results also demonstrate a more coherent structure in the temperature field than that of equivalence ratio. Meanwhile, the analysis of 3D simulation results by POD proves a more coherent structure for the in-cylinder flow regime at top dead center.

\section{ACKNOWLEDGEMENTS}

This research has benefited from the support of the Vehicle, Fuel and Environment Research Institute, University of Tehran. These supports are gratefully 
acknowledged.

\section{REFERENCES}

Bizon, K., G. Continillo. and E. Mancaruso. (2009). POD-based analysis of cycle-to-cycle variations in an optically accessible diesel engine. Proceeding of the combustion institute 32(2), 2809-2816.

Bizon, K., G. Continillo., E. Mancaruso., S. S. Merola. and B.M Vaglieco. (2010). POD based analysis of combustion image in optically accessible engines. Combustion and Flame 157(4), 632-640.

Chen, W., S. Shuai. and J. Wang (2009), A soot formation embedded reduced reaction mechanism for diesel surrogate fuel, Fuel 88(10), 1927-1936.

Chen, H., D. L. Reuss and V. Sick. (2011), Analysis of misfires in a direct injection engine using proper orthogonal decomposition, Experiments in Fluids 51(4), 1139-1151.

Cosadia, I., J Boree., G. Gharnay. and P. Dumont. (2006), Cyclic variation of the swirling low in a diesel transparent engine, Experiments in Fluids 41(1), 115-134.

Cosadia, I., J Boree., G. Gharnay. and P. Dumont. (2007), Coupling time-resolved PIV flowfields and phase-invariant proper orthogonal decomposition for the description of the parameter space in a transparent Diesel engine, Experiments in Fluids 43(2), 357-370.

Druault, P., F. Guibert. and F. Alizon. (2005), Use of proper orthogonal decompositionfor time interpolation from PIV data, Experiments in Fluids 39(6), 1009-1023.

Druault, P. and C. Chaillou. (2007), Use of proper orthogonal decomposition for reconstructing the 3D in-cylinder mean field from PIV data, Comptes Rendus Mécanique 335(1), 42-47.

Fogleman, M., J. L. Lumley., D. Rempfer. and D. Haworth. (2005), Application of proper orthogonal decomposition to datasets of internal combustion engine flows, Journal of Turbulence 5(1), 23-40.

Graciano, V., J. V. C. Vargas and J. C. Ordonez. (2015), Modeling and simulation of diesel, biodiesel and biogas mixtures driven compression ignition internal combustion engines, International Journal of Energy Research 40(1), 100-111.

Heywood, J. B. (1988). Internal combustion engine fundamentals, Singapore: McGrawHill.
Imberdis, O. (2009), Turbulent flow structures induced by an engine intake port, $\mathrm{Ph}$. D. thesis, Magdeburg University, Magdeburg, Germany.

Javaheri, A., V. Esfahanian., A. Salavati-Zadeh. and Darzi M. (2014), Energetic and exergetic analyses of a variable compression ratio spark ignition gas engine, Energy Conversion and Management 88, 739-748.

Karhunen, K. (1946), Zur Spektraltheorie Stochasticher Prozesse, Annales Academiae Scientiarum Fennicae 37(34), 1-7.

Liu, K. and D. C. Haworth. (2011), Development and assessment of POD for analysis of turbulent flow in piston engines, $S A E$ Technical Paper, 2011-01-0830.

Loeve, M. (1946), Functions aleatoire de second ordre, Comptes Rendus de l'Academie des Sciences 84, 195-206.

Lumley, J. L. (1967), The structure of inhomogeneous turbulent flows, In Atmospheric Turbulence and Radio Propagation, Nauka, Moscow.

Pulkrabek, W. W. (1997), Engineering fundamentals of the internal combustion engines, Prentice Hall.

Rakopoulos, C. D., D. C. Rakopoulos. and D. A. Kyritsis. (2003), Development and validation of a comprehensive two-zone model for combustion and emissions formation in a DI diesel engine, International Journal of Energy Research, 27(14), 1221-1249.

Rakopoulos, C. D. and E. G. Giakoumis. (2006), Second law analyses applied to internal combustion engines operation, Progress in Energy and Combustion Science 32(1), 2-47.

Salavati-Zadeh, A., V. Esfahanian., A. Afshari. and M. Ramezani. (2013), A multizone spray and combustion model for formation of polycyclic aromatic hydrocarbons and soot in diesel engines, In SAE-China and FISITA (Eds.), Proceedings of the FISITA 2012 World Automotive Congress, Lecture Notes in Electrical Engineering 190, 1069-1077.

Salavati-Zadeh, A., V. Esfahanian. and H. Akbari (2014), Investigating the effects of fuel flow rate and equivalence ratio on formation of soot in laminar premixed flames of $\mathrm{C} 2$ hydrocarbons using POD technique, Computational Thermal Sciences 6(4), 361367.

Sirovich, L. (1987), Turbulence and dynamics of coherent structures, Quarterly of Applied Mathematics 45(3), 561-590. 